baculovirus DNA can rescue recombinant baculoviruses, provided sufficient flanking DNA is present to overlap the boundaries of the linearized genome. In addition, the use of mutagenized PCR fragments can generate baculovirusbased expression libraries suitable for the direct selection of variants with altered function.

\title{
REFERENCES
}

1.Cadwell, R. and G.F. Joyce. 1992. Random ization of genes by PCR mutagenesis. PCR Methods Appl. 2:28-33.

2.Chen, B. and A.E. Przybyla. 1994. An efficient site-directed mutagenesis method based on PCR. BioTechniques 17:657-659.

3.Kitts, P.A., M.D. Ayres, and R.D. Possee. 1990. Linearisation of baculovirus DNA enhances the recovery of recombinant virus expression vectors. Nucleic Acids Res. 18:56675672.

4.Kitts, P. A. and R.D. Possee. 1993. A method for producing recombinant baculovirus at high frequency. BioTechniques 14:810-817.

5.Luckow, V.A., S.C. Lee, G.F. Barry, and P.O. Olins. 1993. Efficient generation of infectious recombinant baculoviruses by site-specific transposon-mediated insertion of foreign genes into a baculovirus genome propagated in Escherichia coli. J. Virol. 67:4566-4579.

6.Patel, D., K. Nasmyth, and N. Jones. 1992. A new method for the isolation of recombinant baculovirus. Nucleic Acids Res. 20:97104.

7.Vallejo, A.N., R.J. Pogulis, and L.R. Pease. 1994. In vitro synthesis of novel genes: mutagenesis and recombination by PCR. PCR Methods Appl. 4:s123-s130.

Address correspondence to Dr. Susan D.J. Chapple, NERC Institute of Virology, Mansfield Road, Oxford, OX1 3SR, UK. email:sdc@ceh.ac.uk

Received 20 November 2000; accepted 8 March 2001.

Meena Patil, Christine
Chan-Fook, Ian M. Jones ${ }^{1,}$
and Susan D.J. Chapple
NERC Institute of Virology
Oxford
${ }^{1}$ University of Reading
Reading, UK

Meena Patil, Christine Chan-Fook, Ian M. Jones ${ }^{1}$, and Susan D.J. Chapple NERC Institute of Virology Oxford Reading, $U K$

\section{Hybridization to High- Density Filter Arrays of a Brugia malayi BAC Library with Biotinylated Oligonucleotides and PCR Products}

BioTechniques 30:1216-1224 (June 2001)

The development of chemiluminescent detection has provided a rapid, nonradioactive method for positional mapping to cDNA or genomic libraries gridded onto high-density filter arrays $(6,14)$, Southern and northern blotting, plaque lifts, colony hybridizations, and DNA sequencing $(4,5,7,11,12)$. These nonradioactive methods are being used for mapping the genome of Brugia malayi, a parasitic nematode and causative agent of lymphatic filariasis. This disease affects over 120 million people worldwide, with over a billion people at risk from infection from three main species of filarial nematodes $(3,9,15)$. A full description of the project is provided at http://neb.com/fgn/filgen1.html.

More than 22000 expressed sequence tags (ESTs) representing over 8000 different genes have been sequenced from cDNA libraries constructed from various life-cycle stages. For mapping these genes to the $B$. malayi genome, amplified cDNA inserts have been labeled through the incorporation of biotinylated nucleotides by random priming (NEBlot Phototope $^{\mathrm{TM}}$ kit; New England Biolabs, Beverly, MA, USA) and subsequently hybridized to high-density membrane arrays of an 18000 -clone B. malayi BAC library. Positive hybridizations were identified by chemiluminescent detection $(6,10)$. A PCR-based, bacterial artificial chromosome (BAC)-end rescue method has provided additional probes for a chromosomal walking approach. However, the process of preparing suitable probes for hybridization involves multiple steps (i.e., PCR of the DNA insert, restriction digestion to remove vector sequence, purification of the product, and random prime labeling of DNA) and is time consuming. To increase our mapping throughput, we have developed nonradioactive hy- bridization methods with biotinylated oligonucleotides or PCR products generated using biotinylated primers for use as probes. These methods maintain the hybridizaton fidelity and high signal-to-noise ratio observed with probes prepared by random priming.

Oligonucleotides were synthesized at the 40-nM scale using models 392, 394, or Expedite ${ }^{\mathrm{TM}}$ M.O.S.S. DNA synthesizers (Applied Biosystems, Foster City, CA, USA). A biotin-T phosphoramidite and an 18-carbon atom spacer phosphoramidite (both from Glen Research, Sterling, VA, USA) were used in the synthesis of 20-30 oligomers, which have two biotin-labeled Ts separated by the spacer at the $5^{\prime}$ end of the oligonucleotide. Crude syntheses were directly used for all protocols.

PCR was performed, with one or both primers being biotinylated, using AmpliTaq ${ }^{\circledR}$ DNA polymerase (Applied Biosystems). The reactions were purified through QIAquick ${ }^{\mathrm{TM}}$ columns (Qiagen, Valencia, CA, USA) or Microcon ${ }^{\circledR}-50$ columns (Amicon, Danvers, MA, USA).

For the generation of biotin-labeled, BAC-end probes, insert-specific primers based on BAC-end sequences (13) were used in conjunction with a double-biotin-conjugated, vector-specific primer, 2BBACF3 (e.g., 5'-biotin-spacer-biotinGAGTCGACCTGCAGGCATGC-3') or 2BBACR4 (5'-biotin-spacer-biotinTAGGTCACACTATAGAATACTC-3'), to amplify the T7 or SP6 ends, respectively, of inserts cloned into the pBeloBAC11 vector. The insert-specific primers were designed to ensure that the amplified PCR products were between 200 and $500 \mathrm{bp}$. The vector primers, 2BBACR4 and 2BBACF3, are 22 and $23 \mathrm{bp}$ from the vector cloning site, respectively. These short lengths of vector incorporated into the PCR probe did not affect the quality of the subsequent hybridization. Standard PCRs were performed using Taq DNA polymerase (Qiagen) and analyzed by gel electrophoresis to verify the occurrence of a single PCR product. The amplified product was excised from the gel, and the DNA was eluted from the gel slice using an Ultrafree ${ }^{\circledR}$-DNA extraction column (Amicon). The DNA was concentrated into a $40-\mu \mathrm{L}$ volume using a DNA vacuum concentrator (Savant 
Instruments, Holbrook, NY, USA). One microgram was used as a probe for hybridization to the BAC filters.

Biotinylated BAC-end probes were also generated following a modified thermal asymmetric interlaced-PCR (TAIL-PCR) method (8). The method was adapted to amplify end sequences from pBeloBAC11 clones when the insert sequence was unknown. A set of three nested vector primers was designed to each of the SP6 and T7 ends of the pBeloBAC11 vector so that the tertiary primers flanked the cloning

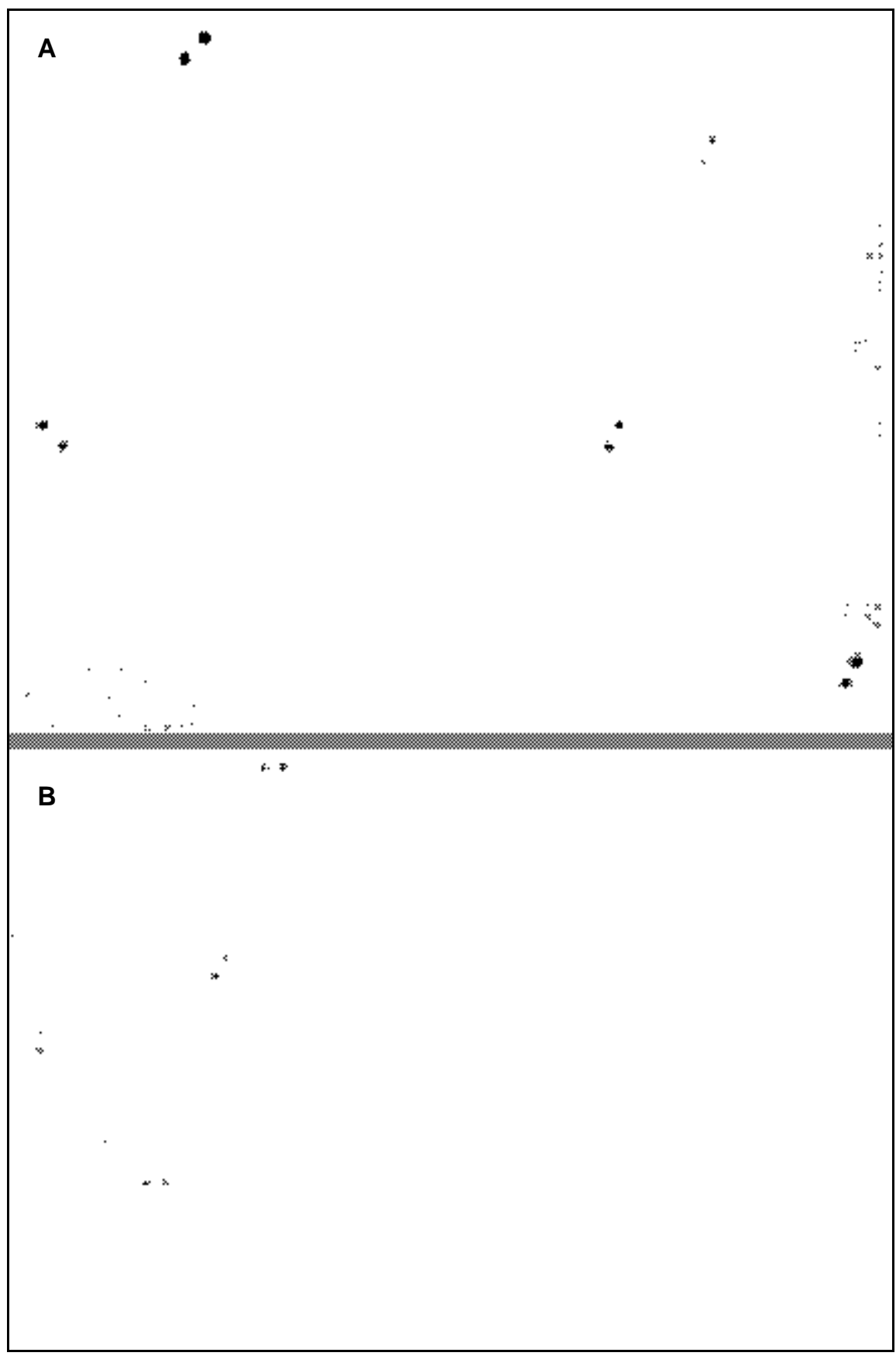

Figure 1. Hybridization to a high-density BAC array. (A) Hybridization using a 22-mer oligonucleotide probe synthesized with two biotin moieties on it and (B) a double-stranded, BAC-end rescue probe synthesized with one double-biotinylated PCR primer, as described in the text. Hybridized probes were detected with the Phototope-Star Detection kit (i.e., streptavidin/alkaline phosphatase/CDP-Star). Positive hybridizations, indicated by double hits within a given square in a pattern consistent with the robotic double spotting of colonies on the filters, were verified as correct by PCR. site. These tertiary primers were double-biotin-labeled at the $5^{\prime}$ end, resulting in biotinylated PCR products that were used as hybridization probes. For the SP6 end of pBeloBAC11, we used the following primers: 5'-CACTCATTAGGCACCCCAGGC-3' (BACR1), 5'-GCCAAGCTATTTAGGTGAC-3'

(BACR3), and 2BBACR4. For the T7 end, we used the following oligonucleotides: 5'-GCGAAAGGGGGATG TGCTGC-3' (BACF1), 5'-GGCGATTAAGTTGGGTAACG-3' (BACF2), and $2 \mathrm{BBACF} 3$. The arbitrary degenerate primer, AD1 (8), worked best among several primers and was used in conjunction with the specific primers previously described.

For TAIL-PCR, BAC clones were grown overnight in $100 \mu \mathrm{L}$ LB plus chloramphenicol $(12.5 \mu \mathrm{g} / \mathrm{mL})$. The cells were pelleted, resuspended in 50 $\mu \mathrm{L}$ sterile Milli- $\mathrm{Q}^{\circledR}$ (Millipore, Bedford, MA, USA) double-distilled water, and heated to $99^{\circ} \mathrm{C}$ for $5 \mathrm{~min}$. Cell debris was pelleted by microcentrifugation for $30 \mathrm{~s}$, and the supernatant was recovered for use as template. The TAIL-PCRs were performed as described (8) with minor modifications. In the primary reaction, $0.2 \mu \mathrm{M}$ vector primer (i.e., BACF1 or BACR1) was used in a $20-\mu \mathrm{L}$ reaction with $2 \mu \mathrm{L}$ template. The secondary and tertiary TAIL-PCRs were also performed as described (8), except that the tertiary PCR used $0.2 \mu \mathrm{M}$ biotinylated vector primer (i.e., 2BBACF3 or 2BBACR4), the reaction size was doubled, and 30 PCR cycles were used to generate an increased amount of product. The tertiary biotinylated PCR product was excised and purified from an agarose gel and used as a hybridization probe.

The $22 \times 22-\mathrm{cm}$ BAC membrane filters, containing over 18000 double-gridded clones on Hybond ${ }^{\circledR}-\mathrm{N}^{+}$(Amersham Pharmacia Biotech, Piscataway, NJ, USA) or Immobilon ${ }^{\mathrm{TM}}-\mathrm{Ny}^{+}$membranes (Millipore), were prepared by Incyte Genomics (St. Louis, MO, USA). For hybridization, each filter was placed in a large plastic tray with a lid (NUNC ${ }^{\mathrm{TM}}$ Bio-Assay Dish; Nalge Nunc International, Rochester, NY, USA) and prehybridized for $1 \mathrm{~h}$ at $55^{\circ} \mathrm{C}$ with $75 \mathrm{~mL} 1$ mM EDTA, 7\% SDS, $0.25 \mathrm{M}$ disodium phosphate, $\mathrm{pH} 7.2(0.5 \mathrm{M}$ disodium phosphate stock $=0.5 \mathrm{M} \mathrm{Na} \mathrm{HPO}_{4}$, 
$\left.0.34 \% \mathrm{H}_{3} \mathrm{PO}_{4}\right)$. Biotinylated oligonucleotides (40 nM synthesis) were resuspended in approximately $1 \mathrm{~mL}$ sterile Milli-Q double-distilled water to a final concentration of $330 \mu \mathrm{g} / \mathrm{mL}$. Typically, $1 \mu \mathrm{g}$ biotinylated oligonucleotide or 1 $\mu \mathrm{g}$ purified biotinylated PCR product was denatured by boiling for $5 \mathrm{~min}$, placed on ice for $5 \mathrm{~min}$, briefly microcentrifuged at $4{ }^{\circ} \mathrm{C}$, and then added to the prehybridization solution in the Bio-Assay dish. Following hybridization with gentle rocking at $55^{\circ} \mathrm{C}$ overnight, the probe solutions were removed and stored at $-20^{\circ} \mathrm{C}$ for reuse if required. Probes have been stored at $-20^{\circ} \mathrm{C}$ for over a year and reused several times, by boiling the entire $75 \mathrm{~mL}$ hybridization sample for $5 \mathrm{~min}$ before cooling to $55^{\circ} \mathrm{C}$ and adding it to the filters.

The filter was then washed twice in the Bio-Assay dish with gentle rocking for 5 min each at room temperature in $200 \mathrm{~mL} 2 \times$ standard saline citrate (SSC) $(0.3 \mathrm{M} \mathrm{NaCl}, 0.03 \mathrm{M}$ sodium citrate, $\mathrm{pH} 7.0$ ), and $0.1 \%$ SDS. Then, we performed two 15-min stringency washes each at $55^{\circ} \mathrm{C}$, rocking in $80 \mathrm{~mL}$ $1 \times$ SSC, and $1 \%$ SDS (12) in the covered dish. The hybridized probes were then detected using the Phototope ${ }^{\mathrm{TM}}$ Star Detection kit (New England Biolabs) (10), as modified in Table 1. In steps $1-6$, each 5-min incubation was performed with rocking, followed by pouring off and discarding the used solution. All plasticware was rinsed with sterile Milli-Q double-distilled water before use.

For the $22 \times 22-\mathrm{cm}$ membrane filter, the volumes used were:

1. A wash with $100 \mathrm{~mL}$ blocking solution [5\% SDS, $25 \mathrm{mM}$ sodium phosphate $\left(17 \mathrm{mM} \mathrm{Na}_{2} \mathrm{HPO}_{4}, 8 \mathrm{mM}\right.$ $\left.\left.\mathrm{NaH}_{2} \mathrm{PO}_{4}\right), \mathrm{pH} 7.2,125 \mathrm{mM} \mathrm{NaCl}\right]$

2. Addition of $75 \mathrm{~mL}$ streptavidin solution $(75 \mu \mathrm{L}$ stock solution in $75 \mathrm{~mL}$ blocking solution; final concentration of streptavidin is $1.0 \mu \mathrm{g} / \mathrm{mL}$ )

3. Four washes of $200 \mathrm{~mL}$ each with wash solution I $[0.5 \%$ SDS, $2.5 \mathrm{mM}$ sodium phosphate $\left(1.7 \mathrm{mM} \mathrm{Na}_{2} \mathrm{HPO}_{4}\right.$, $\left.0.8 \mathrm{mM} \mathrm{NaH}_{2} \mathrm{P} \mathrm{O}_{4}\right), 12.5 \mathrm{mM} \mathrm{NaCl}$; a 1:10 dilution of blocking solution]

4. Addition of $75 \mathrm{~mL}$ biotinylated alkaline phosphatase $(75 \mu \mathrm{L}$ stock solution in $75 \mathrm{~mL}$ blocking solution; final concentration of biotinylated alkaline phosphatase is $0.5 \mu \mathrm{g} / \mathrm{mL}$ )
5. A rinse of $200 \mathrm{~mL}$ blocking solution

6. Four washes of $200 \mathrm{~mL}$ with wash solution II (10 mM Tris-HCl, $\mathrm{pH}$ 9.5, $10 \mathrm{mM} \mathrm{NaCl}, 1 \mathrm{mM} \mathrm{MgCl} 2)$

7. Addition of diluted CDP-Star ${ }^{\mathrm{TM}}$ reagent $(72 \mathrm{~mL}$ sterile Milli-Q doubledistilled water, $3 \mathrm{~mL} 25 \times$ CDP-Star diluent, and $300 \mu \mathrm{L}$ CDP-Star; final dilution of 1:250 of the detection reagent)

All buffers and wash solutions for steps $1-6$ in the protocol and for probe stripping are available in multi-liter quantities (American Bioanalytical, Natick, MA, USA).

Because the reactions and washes were performed in dishes, multiple-filter detections can be performed simultaneously by stacking the dishes on a shaking rocker (e.g., steps 1-6). Step 7 was performed without shaking, after ensuring that the membrane is thoroughly covered by the detection solution. The filters were then wrapped in plastic wrap, and excess detection reagent was removed by rolling a pipet across the wrapped filter. The filter was film (Eastman Kodak, New Haven, CT, USA) from $45 \mathrm{~s}$ to $15 \mathrm{~min}$. The film was developed in a Kodak M35A Automated Developing Processor.

After the completion of the chemiluminescent detection, probes were then exposed to Kodak ${ }^{\circledR}$ BioMax-MS

stripped from the membranes to prepare for subsequent hybridizations. Filters were first rinsed in sterile Milli-Q double-distilled water before being incubated in $80 \mathrm{~mL} 0.4 \mathrm{~N} \mathrm{NaOH}, 0.1 \%$ SDS at $70^{\circ} \mathrm{C}$ for $30 \mathrm{~min}$ in the Bio-Assay dishes with lids. The solutions were decanted, and the filters were rinsed in $200 \mathrm{~mL} 0.2 \mathrm{M}$ Tris- $\mathrm{HCl}$ and $0.1 \times \mathrm{SSC}$, $\mathrm{pH} 7.5$, for $30 \mathrm{~min}$ at room temperature. All steps in this stripping process were performed while gently rocking the dishes. The membranes were then either reprobed or stored in plastic wrap at $-20^{\circ} \mathrm{C}$. We have successfully performed up to 15 stripping and reprobings on each of these.

Figure 1 presents lumigrams using the described protocols. Positive hybridizations are indicated by double "hits" within a given square, in a pattern consistent with the robotic double spotting of colonies on the filters. Figure 1A presents results from a hybridization to a high-density BAC array using an oligonucleotide synthesized with two biotin moieties on it and subsequently detected with the Phototope-Star Detection kit. Figure 1B shows results using a PCR product generated as a BAC-end rescue probe synthesized with one double-biotinylated primer and similarly detected. In each example, the doublebiotinylated product provides sufficient

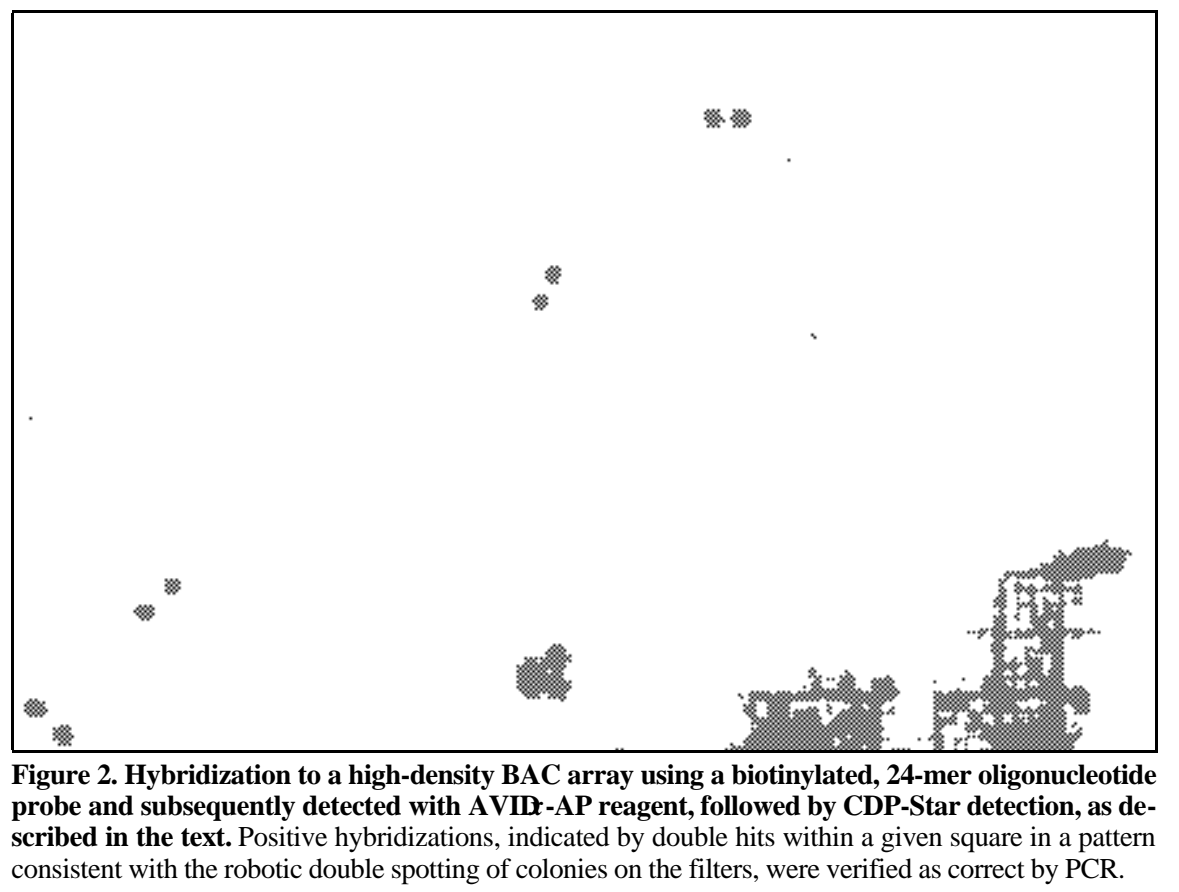


signal in the context of a low background, using the described protocols. Essentially all biotinylated oligonucleotides and biotinylated PCR products gave positive hits to the library in correct positions, from hundreds of hybridizations. Among these, there are occasional instances of "false positives". Because of the double gridding of clones onto the filters, single positive spots or positives inconsistent with the patterns produced by the robot can im mediately be discounted. The remainder are usually caused by cross hybridizations with the probes and can be eliminated by PCR verification of the potential positive clones, increased stringency of the hybridization reaction, or, if necessary, by the synthesis of a new biotinylated oligonucleotide. For our mapping experiments, we have routinely used PCR to verify all of our positive hybridizations.

BAC-end sequencing is now a fairly standard procedure in many laboratories. Perhaps the simplest approach to generating biotinylated probes for chromosome walking is to synthesize double-biotin-labeled oligonucleotides, based on the sequence of the insert DNA in the BAC vector. However, when the $\mathrm{BAC}$-end sequence is unknown, endrescue methods can be used to generate biotinylated probes. This approach may be more appropriate for yeast artificial chromosome (YAC) cloning in which direct end sequencing is not feasible. For other applications, biotinylated PCR products can be generated using a com mon, biotinylated, vector-specific oligonucleotide as one of the PCR primers. Using whichever method might be best applied to a particular project, biotinylated oligonucleotides provide a set of potentially useful reagents for experiments from hybridization mapping to high-density arrays.

As we developed the methods for high-density filter hybridization with biotinylated oligonucleotides and biotinylated PCR products, we also investigated other parameters of the process. Biotinylated oligonucleotides of 20-30 nucleotides in length appear to work as well as hybridization probes and PCR primers. Several other hybridization buffers were also successfully used $(1,2)$. In each case, the addition of sonicated salmon or herring sperm DNA in the prehybridization solution did not improve the signal-tonoise ratio, and they were eliminated from the hybridization solutions. Formamide hybridization buffers were not tried. A range of oligonucleotide probe amounts $(1-10 \mu \mathrm{g})$ was used in attempts to further strengthen the signal. An increase in probe amount did not increase signal strength under the conditions used, suggesting that the $1-\mu \mathrm{g}$ probe was sufficient to saturate the available binding sites on the BAC membranes of this size and composition. Using one or two biotinylated oligonucleotides as primers during PCR made little difference in the resulting signal intensity.

We observed an additional improvement when the last step (step 7) was not accompanied by rocking. This improvement is presumed to be due to a reduction in the movement of the precipitated detection reagent, after dephosphorylation of the CDP-Star reagent, away from the site of the biotin/streptavidin/alkaline phosphatase complex.

We have also investigated the use of preformed, conjugated streptavidin/alkaline phosphatase reagents to combine steps $2-4$ in the above procedure. The best results were obtained with AVIDx$\mathrm{AP}^{\mathrm{TM}}$ (Tropix, Bedford, MA, USA) at a dilution of 1:10000 in blocking buffer (described in step 1), which provided high signal and very low background (Figure 2). This procedure worked for probes that were biotinylated dsDNA products (i.e., including PCR products labeled by random priming or with biotinylated primers). For use with biotinylated oligonucleotide probes, the AVIDx-AP appears to give the best results when diluted 1:50 000 in conjunction with a 1:100 dilution of the CDPStar reagent.

Other techniques, such as alkaline phosphatase-conjugated oligonucleotides and PCR products (e.g., Gene Images $^{\mathrm{TM}}$; Amersham Pharmacia Biotech) and incorporation methods (e.g., digoxigenin, DIG-System; Roche Molecular Biochemicals, Indianapolis, IN, USA) form the basis for future studies on high-density filter arrays.

We have extended the use of nonradioactive hybridization and chemiluminescent detection procedures on highdensity filter arrays, using double- biotin-labeled oligonucleotides, either directly or as primers for PCR. These methods have allowed the nonradioactive mapping of ESTs and BAC-end sequences to cDNA and BAC high-density filters, as part of the Filarial Genome Project. The procedure is robust, and no major equipment, such as CCD cam eras or scanners, is required.

\section{REFERENCES}

1.Church, G. and S. Kieffer-Higgins. 1988. Multiplex DNA sequencing. Science 240:185188.

2.Cornish, E., S. Beckham, and J. Maddox. 1998. Southern hybridization revisited: probe target DNA interaction is affected by the choice of hybridization buffer. BioTechniques 25:949-953.

3.Cox, F.E.G. 2000. Elimination of lymphatic filariasis as a public health problem. Parasitol. Today 16:135.

4.Creasey, A., L. D'Angio, Jr., T.S. Dunne, C. Kissinger, T. O'Keeffe, H. Perry-O'Keefe, L.S. Moran, M. Roskey et al. 1991. Application of a novel chemiluminescence-based DNA detection method to single-vector and multiplex DNA sequencing. BioTechniques 11:102-109.

5.Dubitsky, A., J. Brown, and H. Brandwein. 1992. Chemiluminescent detection of DNA on nylon membranes. BioTechniques 13:392400.

6.Guiliano, D., M. Ganatra, J. Ware, J. Parrot, J. Daub, L. Moran, H. Brennecke, J.M. Foster et al. 1999. Chemiluminescent detection of sequential DNA hybridizations to high-density, filter-arrayed cDNA libraries: a subtraction method for novel gene discovery. BioTechniques 27:146-152.

7.Kincaid, R.L. and M.S. Nightingale. 1988. A rapid non-radioactive procedure for plaque hybridization using biotinylated probes prepared by random primed labeling. BioTechniques $6: 42-50$.

8.Liu, Y.-G. and R.F. Whittier. 1995. Thermal asymmetric interlaced PCR: automatable am plification and sequencing of insert end fragments from P1 and YAC clones for chromosome walking. Genomics 25:674-681.

9.Michael, E. and D. Bundy. 1997. Global mapping of lymphatic filariasis. Parasitol. Today 13:472-476.

10.New England Biolabs, Inc. 1997. Phototope ${ }^{\circledR}$-Star Detection Manual. New England Biolabs, Inc., Beverly, MA, USA.

11.Sheffield, J.S., W.H. Benjamin, and L.S. McDaniel. 1992. Detection of DNA in Southern blots by chemiluminescence is a sensitive and rapid technique. BioTechniques 12:836840.

12.Tumolo, A., Q. Nguyen, F. Witney, O. Murphy, C. Martin, J. Voyta, and I. Bronstein. 1995. Detection on membranes with alkaline phosphatase-labeled probes and chemiluminescent CSPD ${ }^{\mathrm{M}}$ substrate, $\mathrm{p}$ 145-154. In L. Kricka (Ed.), Nonisotopic Probing, Blotting 
and Sequencing. Academic Press, New York.

13.Ware, J., L. Moran, C.-L. Lin, and B. Slatko. 2001. Implementation of automation in a small scale DNA sequencing core facility. J. Biomol. Techniques 11:151-154.

14. Weintraub, D., P. Ularch, and J. Mullet. 1998. Screening BAC high density filters with Southern-StarT ${ }^{\mathrm{T}}$. Luminescence Topics 6:3-4.

15.Williams, S.A., M. Lizotte-Waniewski, J. Foster, D. Guiliano, J. Daub, A. Scott, B. Slatko, and M. Blaxter. 2000. The filarial genome project: analysis of the nuclear, mitochondrial and endosymbiont genomes of $\mathrm{Bru}$ gia malayi. Int. J. Parasitol. 30:411-419.

We thank Roger Knott, John Buswell, and Nancy Considine at the NEB Organic Synthesis Core Facility for aid in design and synthesis of oligonucleotides. We also thank Alan Scott and Anthony Underwood for helpful discussions on approaches described in this manuscript and comments on earlier drafts and Ray Judware (Tropix) for helpful discussions concerning the AVIDxAP reagent. We also acknowledge Ipswich High School (Ipswich, MA, USA) for their interest and support in enabling Jessica Ingram and Matthew Swan to join our laboratory as part of their senior honors student intern program. We thank Don Comb for encouragement and support of this project. Financial support is acknowledged to WHO/TDR/UNDP/World Bank, Medical Research Council, The Wellcome Trust, and to internal funding from NEB. Address correspondence to Dr. Barton Slatko, Molecular Parasitology Group, New England Biolabs, Inc., 32 Tozer Road, Beverly, MA 01915,USA.e-mail:dnaseq@neb.com

Received 18 December 2000; accepted 29 March 2001.

\section{J.M. Foster, I.H. Kamal, J. Daub', M.C. Swan, J.R. Ingram, M. Ganatra, J. Ware, D. Guiliano', A. Aboobaker'1, L. Moran, M. Blaxter ${ }^{1}$, and B.E. Slatko \\ New England Biolabs \\ Beverly, MA, USA \\ 'University of Edinburgh Edinburgh, UK}

\section{Enhanced Capture of Small Histidine-Contain- ing Polypeptides on Membranes in the Presence of $\mathrm{ZnCl}_{2}$}

BioTechniques 30:1224-1230 (June 2001)

Small hydrophilic polypeptides and proteins can be difficult to capture on membranes designed for western blot analysis using standard blotting protocols $(2,6,10)$. The use of charged membranes, such as polyvinylidene difluoride (PVDF), and membranes with small pore sizes can improve capture, but, in some cases, even these mem branes when used with standard transfer protocols do not give satisfactory results. We report a transfer protocol that results in the improved capture of three small histidine-containing polypeptides and is likely to be of use with other histidine-containing proteins, including proteins with histidine tags.

In the course of studying the $8-\mathrm{kDa}$ 84-6 nodulin polypeptide from actinorhizal Alnus $(3,11)$, we observed that immunostaining on western blots, if present at all, was more intense on the backside of the membrane than on the side facing the gel from which proteins were transferred. In subsequent experiments, Coomassie ${ }^{\circledR}$ Brilliant Blue R250 (Bio-Rad Laboratories, Hercules, CA, USA) staining of the Whatman ${ }^{\circledR}$ 3MM blotting papers (Whatman, Clifton, NJ, USA) used in semidry transfer revealed that most of the protein had transferred through the membrane to the blotting papers. Attempts at quantitative capture of the polypeptide on membranes by adjusting transfer buffer composition ( $\mathrm{pH}$, SDS, methanol, continuous vs. discontinuous buffers), blotting times and amperage, as well as using the lowest pore size membranes commercially available (0.1- $\mu \mathrm{m}$ nitrocellulose, 0.2- $\mu \mathrm{m}$ PVDF, and $0.2-\mu \mathrm{m}$ nylon), were not successful. A search of the literature led us to papers by McKeon and Lyman (8) and Mizzen et al. (9) that reported enhanced retention of calmodulin and metallothionine, respectively, in the presence of $2 \mathrm{mM} \mathrm{CaCl}_{2}$. In light of these results, we tested the ability of $\mathrm{CaCl}_{2}$ to improve capture on membranes of nodulin 84-6. Addition of $2 \mathrm{mM} \mathrm{CaCl}_{2}$ to transfer buffers did not result in quantitative capture, nor did the presence of $\mathrm{MgCl}_{2}$ or $\mathrm{MnCl}_{2}$ in transfer buffers improve capture on any membranes tested.

Nodulin 84-6 is a histidine-containing polypeptide that binds the follow ing divalent cations: $\mathrm{Ni}^{+2}, \mathrm{Zn}^{+2}, \mathrm{Co}^{+2}$, $\mathrm{Cu}^{+2}, \mathrm{Cd}^{+2}$, and $\mathrm{Hg}^{+2}$, but not $\mathrm{Ca}^{+2}$, $\mathrm{Mg}^{+2}$, and $\mathrm{Mn}^{+2}$ (data not shown). Therefore, we tested the ability of these divalent cations to enhance the capture of nodulin 84-6 on 0.2 - and $0.45-\mu \mathrm{m}$ PVDF, 0.1 - and $0.45-\mu \mathrm{m}$ nitrocellulose, and 0.2 - and $0.45-\mu \mathrm{m}$ nylon membranes (Schleicher \& Schuell, Keene, $\mathrm{NH}$, USA). The addition of $2 \mathrm{mM}$ $\mathrm{ZnCl}_{2}, \mathrm{CdCl}_{2}$, and $\mathrm{HgCl}_{2}$ separately to transfer buffer consistently enhanced the capture of nodulin 84-6 on $0.2-\mu \mathrm{m}$ PVDF, $0.1-\mu \mathrm{m}$ nitrocellulose, and $0.2-\mu \mathrm{m}$ nylon membranes. Only a faint protein band of 84-6 was observed on PVDF and nitrocellulose membranes in transfers without these metals. When using nylon membranes under the same conditions without added metal, protein capture was undetectable with im munoblotting procedures. These same results were observed when a second small histidine-containing nodulin from Alnus, nodulin 164-20, was transferred with or without metals in the transfer buffer. Figure 1 shows the effect of the presence of $2 \mathrm{mM} \mathrm{ZnCl} 2$ in the transfer buffer on the capture of nodulins 84-6 and 164-20 on PVDF, nylon, and nitrocellulose membranes. Density scans of the blots indicate that from 4 to 10 times more protein is captured on the membranes when electroblotting is done in the presence of 2 $\mathrm{mM} \mathrm{ZnCl} 2$. Addition of cadmium and mercury to transfer buffers results in equal enhancement of capture, but because of toxicity and disposal problems associated with these metals, we do not recommend their use in electroblotting.

In an effort to determine if the addition of $\mathrm{ZnCl}_{2}$ to transfer buffers had a wider applicability, we applied the technique to another small difficult-tocapture histidine-containing polypeptide, the $A \beta$ amyloid peptide (fragment 1-40) associated with Alzheimer's disease, which we had previously found passed through nylon, nitrocellulose, 